\title{
Identification and characterization of a non- conventional CD45 negative perivascular macrophage population within the mouse brain.
}

\section{Carole Siret}

CIML

Max van Lessen

WWU Münster

Hyun-Woo Jeong

Max Planck Institute for Molecular Biomedicine, Department of Tissue Morphogenesis, 48149 Münster, Germany https://orcid.org/0000-0002-6976-6739

\section{Shuaiwei Wang}

CIML

Milesa Simic

CIML

\section{Lauriane de Fabritus}

CIML

Aurelie Tchoghandjian

Aix-Marseille University

Mathieu Fallet

CIML

\section{Sandrine Sarrazin}

CIML

\section{Michael Sieweke}

Centre d'Immunologie de Marseille - Luminy, Aix Marseille University, Centre National de la Recherche Scientifique, Institut National de la Santé et de la Recherche Médicale/Max-Delbr https://orcid.org/00000002-3228-9537

\section{Ralf Stumm}

Jena University Hospital https://orcid.org/0000-0002-9458-5526

\section{Ralf Adams}

Max Planck Institute for Molecular Biomedicine https://orcid.org/0000-0003-3031-7677

\section{Stefan Merker}

WWU Münster

Friedemann Kiefer 
Max Planck Institute for Molecular Biomedicine Department, Vascular and European Institute for Molecular Imaging, University of Munster, Munster

Serge van de Pavert ( $\square$ vandepavert@ciml.univ-mrs.fr)

CIML https://orcid.org/0000-0002-7147-4380

\section{Article}

Keywords: brain vasculature, lymphatic vasculature, inflammation, drainage, whole mount imaging, stroke, photothrombosis, perivascular macrophages

Posted Date: May 19th, 2021

DOI: https://doi.org/10.21203/rs.3.rs-479980/v1

License: (c) (1) This work is licensed under a Creative Commons Attribution 4.0 International License. Read Full License 
Identification and characterization of a non-conventional CD45 negative perivascular macrophage population within the mouse brain.

C. Siret ${ }^{1}$, M. van Lessen ${ }^{2}$, H. W. Jeong ${ }^{3}$, S. Wang ${ }^{1}$, M. Simic ${ }^{1}$, L. de Fabritus ${ }^{1}$, A. Tchoghandjian ${ }^{4}$, 5 M. Fallet ${ }^{1}$, S Sarrazin ${ }^{1}$, M.H. Sieweke ${ }^{1,5}$, R. Stumm ${ }^{6}$, R. H. Adams ${ }^{3}$, S. Schulte-Merker ${ }^{2}$, F. Kiefer ${ }^{3,8}$,

6 S.A. van de Pavert ${ }^{1, *}$

$8{ }^{1}$ Aix-Marseille Univ, CNRS, INSERM, Centre d'Immunologie de Marseille-Luminy (CIML),

9 Marseille, France

$10{ }^{2}$ Institute for Cardiovascular Organogenesis and Regeneration, Faculty of Medicine, WWU Münster,

11 Mendelstrasse 7, 48149 Münster, Germany

$12{ }^{3}$ Max Planck Institute for Molecular Biomedicine, Münster, Germany.

$13{ }^{4}$ Aix-Marseille Univ, CNRS, INP, Inst Neurophysiopathol, Marseille, France.

$14{ }^{5}$ Center for Regenerative Therapies Dresden (CRTD), Technische Universität Dresden, 01307

15 Dresden, Germany

$16{ }^{6}$ Institute of Pharmacology and Toxicology, Jena University Hospital, Jena, Germany

$17{ }^{8}$ European Institute for Molecular Imaging - EIMI, Westfälische Wilhelms-Universität Münster,

18 Münster, Germany.

19 * Correspondence:

20 Serge van de Pavert; vandepavert@ciml.univ-mrs.fr

21 Keywords: brain vasculature, lymphatic vasculature, inflammation, drainage, whole mount imaging, stroke, photothrombosis, perivascular macrophages 


\section{ABSTRACT}

25 Perivascular macrophages (pvM) are closely associated with cerebral vasculature and play an essential 26 role in drainage of the brain and regulation of the immune response. Here, using reporter mouse models and immunofluorescence on sections and whole brain, flow cytometry and single cell sequencing, we identify a Lyve1 ${ }^{+}$brain perivascular population lacking classical macrophage markers such as CD45 and $\mathrm{Cx} 3 \mathrm{cr} 1$. We named the new non-conventional CD45 negative perivascular macrophages pvM2. These cells have a similar location, morphology and phagocytic function as conventional pvM. The pvM2 are not derived from hematopoietic stem cells, as they are negative in the $V a v^{t d T}$ lineage tracing model. They increase in number after photothrombotic induced stroke established by flow cytometry and 3D immunofluorescence analysis. Since CD45 negative cells were typically excluded from macrophage studies, the presence of pvM2 has been previously missed and their role is of importance to assess in the brain disease models.

\section{INTRODUCTION}

The central nervous system (CNS) has long been considered immune privileged, devoid of immune cells other than microglia and without classical lymphatic vessels. The re-discovery of the dural lymphatic network highlights a route for the drainage of the brain to the periphery ${ }^{1-6}$. How drainage from the CNS parenchyma occurs towards lymphatics and draining lymph nodes is unknown, but there are indications this happens via the foramen at the base of the skull ${ }^{3}$.

Besides microglia, several myeloid populations have now been characterized and shown to be essential for brain homeostasis and in brain diseases ${ }^{7}$. These myeloid populations include the so-called nonparenchymal or border macrophages which can be classified in the perivascular, subdural meningeal and choroid plexus macrophages. These macrophages are established during development by embryonic precursors derived from yolk sac precursors and are not replaced by blood monocytes during adulthood, except for the choroid plexus macrophages ${ }^{8}$. Specifically, perivascular macrophages (pvM) are located in the perivascular space of the blood vessel, delimited by the vascular basement membrane of blood vessels and glial basement membranes ${ }^{9}$. The pvM have been shown to be involved in many processes within the CNS. Early studies demonstrated that pvM are capable of scavenging molecules injected into the cerebral ventricles. They contribute to the blood brain barrier function and mediate the up-take of macromolecules ${ }^{10-12}$, participate in immune regulation ${ }^{13}$. They are involved in a wide variety of brain related disorders such as cerebrovascular and neurocognitive functions in

55 hypertension ${ }^{14}$, brain infections, immune activation, Alzheimer's disease ${ }^{15,16}$ and multiple sclerosis 
$56{ }^{17,18}$, suggesting that they are a key component of the brain-resident immune system and involved in

57 clearing or draining of the CNS.

58 Lyve1 is notably expressed on lymphatic endothelial cells (LEC) and macrophages, but not on 59 microglia. Specifically, distinct macrophage populations lining the blood vessels within the periphery

60 have been characterized as Lyve $1^{\text {hi }}$ and Lyve $1^{\text {lo }}{ }^{19}$. In the mouse brain, myeloid cells are a 61 heterogeneous group of cells localized in specific niches and include parenchymal microglia and non62 parenchymal pvM ${ }^{7,20}$. Previously, brain pvM were described to be $\mathrm{CD}^{+} 5^{+}$and were shown to express 63 canonical macrophage markers such as fractalkine receptor ( $\mathrm{Cx} 3 \mathrm{cr} 1)$, colony-stimulating factor1 64 receptor (Csf-1R), CD206 and Iba-1, but also the prototypic macrophage markers CD11b and F4/80 $65 \quad 7,8$.

66 Recent studies highlighting Lyve $1^{\text {hi }}$ and Lyve $1^{\text {lo }}$ pvM ${ }^{19}$, peripheral nervous system and specific 67 border-associated macrophages ${ }^{21,22}$ have shown that the macrophage population within the nervous 68 system is heterogenous. However, all these studies have excluded CD45- cells. In the current study, 69 using different reporter- and fate-mapping mouse models and employing both (whole-mount) 70 immunofluorescence as well as flow-cytometry and single cell sequencing, we observed a previously 71 uncharacterized $\mathrm{CD} 45^{-} \mathrm{CX} 3 \mathrm{CR} 1^{-} \mathrm{PU} .1^{-} \mathrm{Lyve} 1^{+} \mathrm{F} 4 / 80^{+}$cell population within the mouse brain. We 72 established that these cells were not lymphatic endothelial cells, but non-conventional pvM lining 73 blood vessels, having a similar morphology as conventional pvM. Functionally, they were able to 74 phagocytose macromolecules injected within the ventricle. During photothrombotic-induced stroke, $75 \mathrm{CD}^{-} \mathrm{pvM}$ increased similar as conventional pvM. Hence, our data characterize a previously 76 overlooked $\mathrm{CD}^{-} \mathrm{pvM}$ cell population, which we name non-conventional $\mathrm{CD}^{-} 5^{-}$perivascular 77 macrophages (pvM2). 
RESULTS

79

80

\section{Identification of a $\mathrm{Lyve}^{+} \mathrm{CX} 3 \mathrm{CR} 1^{-}$cell population in the brain parenchyma}

Using whole-mount immunofluorescence of the superior and inferior cortex and cerebellum, we identified Lyve ${ }^{+}$cells that did not form lymphatic luminized vessels (Fig. 1A-D, whole brain in Video 1 and maximum intensity projection in Supplementary Fig. 1A). Their cellular morphology depended on their location. Within the superior cortex (Fig. 1A) they were small and spread, similar as in the pia mater. Within the inferior cortex, these cells were elongated and more stretched, as they were also within the hippocampus (Fig. 1B-C). In the olfactory bulb, near the cribriform plate, where cerebrospinal fluid (CSF) lymphatic drainage occurs from the sub-arachnoid space into nasal lymphatics, Lyve $1^{+}$cells were also found to be long and stretched (Fig. 1D).

In $\mathrm{Cx} 3 \mathrm{crl}^{\mathrm{GFP/+}}$ and Spi ${ }^{G F P /+}$ (gene encoding PU.1) mouse brain sections, we identified conventional $\mathrm{Lyve}^{+} \mathrm{CX} 3 \mathrm{CR} 1^{+} \mathrm{F} 4 / 80^{+} \mathrm{Iba}^{+}$pvM (white arrows in Fig. 1). In addition, we observed a Lyve $1^{+} \mathrm{CX} 3 \mathrm{CR}^{-}$population expressing F4/80 and Iba1 (Fig. 1E, F) but lacking classical hematopoietic and other macrophage markers such as CD45, Csf1-R and PU.1 (red arrows in Fig. 1E-I), which has not been described before. We confirmed the lack of CX3CR1 and PU.1 on sections using an anti-GFP (Supplementary Fig. 1B,C). Furthermore, these atypical Lyve ${ }^{+} \mathrm{CX} 3 \mathrm{CR} 1^{-}$cells lacked CD163, CD206, MHCII and CD11b expression (Supplementary Fig. 1D-G). The pvM markers expressed are summarized in Table 2.

\section{Lyve1 $^{+}$CX3CR1- cells are non-conventional $\mathrm{CD}^{-} 5^{-}$perivascular macrophages (pvM2)}

In the light of the recent re-discovery of lymphatic vessels in the meningeal compartment ${ }^{1,6,32}$, we investigated whether the Lyve $1^{+} \mathrm{CX} 3 \mathrm{CR} 1^{-} \mathrm{CD} 45^{-}$population in the parenchyma of adult mice was of lymphatic origin. We observed Lyve $1^{+}$cells in very close association with blood vessels (Fig. 2A-D, Video 2) and located outside of a structure called "pia mater cul de sac" (Fig. 2B-D). The pia mater formed invaginations into the brain parenchyma, visualized by Podoplanin ${ }^{9}$, surrounding arterioles within the cortex. We observed that the Podoplanin ${ }^{+}$pia was located between the vascular endothelium and the Lyve ${ }^{+}$cells (Fig. 2B-D, Supplementary Fig. 2A). We also observed that the Lyve1 ${ }^{+}$cells were positioned within the Laminin $\gamma 1^{+}$endothelial cell basement membrane within the perivascular space (Fig. 2E-F). This compartment is defined by the vascular basement membrane on the abluminal side of the vessel wall and by the glia limitans basement membrane on the parenchymal side ${ }^{33}$. We observed that Lyve1 ${ }^{+}$cells did not express CD31 (Fig. 2A-H), Podoplanin (Fig. 2B-D), VEGFR3 (Fig. 2G-H) or master-regulator for lymphatic endothelial identity Prox1 (Fig. 2I-L), ruling out a lymphatic identity. We verified non-lymphatic phenotype using ProxlmOrange2 (Supplementary Fig. 2B-C) and 
$111 \operatorname{Prox}^{\text {CreErt2}}$;Rosa26 ${ }^{t d T}$ (Supplementary Fig. 2D) reporter mouse models 24,34. We analyzed the

$112 \operatorname{Prox}^{\text {CreErt2 }}$;Rosa $26^{t d T}$ brain 2 weeks after Tamoxifen injection and did not observe Prox $1^{+}$lymphatic

113 endothelial cells, ruling out any LEC identity within the brain parenchyma (Supplementary Fig. 2B).

114 While the dura mater contains lymphatic vessels, no conventional lymphatics have been described

115 within the parenchyma nor the leptomeninges. Similarly, while we did not observe luminized

116 lymphatic vessels within the pia mater, we did find single Lyve $1^{+}$Prox $1^{+}$lymphatic endothelial cells in

117 the pia mater (Supplementary Fig. 2B-C) as was also recently described in mammals ${ }^{35}$.

118 To further rule out astrocyte, glial or fibroblast identity, we analyzed immunofluorescence staining for

119 Aquaporin-4 (AQP4) and GFAP (Supplementary Fig. 2D), ER-TR7 (Supplementary Fig. 2E),

120 PDGFR $\beta$ (Supplementary Fig. 2F) and observed these cells to be negative for all. Furthermore, we

121 excluded neural crest cell origin using the $W n t 1^{C r e}$; Rosa $26^{t d T}$ reporter mouse model (Supplementary 122 Fig. 2G).

123

124 We confirmed the presence of the Lyve $1^{+} \mathrm{CD} 45^{-}$populations at different stages during life by flow 125 cytometry. Using $C x 3 \mathrm{crl}^{G F P}$ brain parenchyma cell suspension devoid of meninges, conventional pvM 126 were identified as Lyve ${ }^{+} \mathrm{CD} 45^{\text {int/high }}$ while microglia were excluded as they are Lyve1 ${ }^{-}$(gating strategy 127 in Supplementary Fig. 3A). These Lyve $1^{+} \mathrm{CD} 45^{-}$cells also lacked CX3CR1, confirming IF section 128 stainings (Fig. 1). After 2 days after birth (P2) we observed a Lyve $^{+} \mathrm{CD} 45^{-} \mathrm{CX} 3 \mathrm{CR} 1^{-} \mathrm{F} 4 / 80^{+}$ 129 population which peaked at P14 and P21(Fig. 3A-D). Subsequently, we observed lowered numbers in 130 adult and 1-year old brain parenchyma (Fig. 3E-F, absolute Lyve ${ }^{+} \mathrm{CD} 45^{-} \mathrm{CX} 3 \mathrm{CR} 1^{-} \mathrm{F} 4 / 80^{+}$cell 131 numbers in Fig. 3G). Also in the Spi1 ${ }^{G F P}$ (encoding PU.1) brain parenchyma we observed a CD45132 PU. $1^{-} \mathrm{F} 4 / 80^{+}$population, next to the conventional CD45 int/high populations (Supplementary Fig. 3C), 133 albeit less $\mathrm{Lyve}^{+} \mathrm{CD} 45^{-} \mathrm{PU} .1^{-} \mathrm{F} 4 / 80^{+}$cells were present compared to the $\mathrm{Lyve} 1^{+} \mathrm{CD} 45^{-} \mathrm{CX} 3 \mathrm{CR} 1^{-}$ 134 population observed in the $C x 3 c r 1^{G F P}$ brain parenchyma.

136 We confirmed absence of Ptprc (encoding CD45) expression within the Lyve $1^{+} \mathrm{CD}_{4} 5^{-}$cells by sorting 137 this population and determination of Ptprc mRNA levels by qPCR and observed no Ptprc expression 138 within the Lyve $1^{+} \mathrm{CD} 45^{-}$population (Fig. 4A, sorting strategy in Supplementary Fig. 4A). To further 139 characterize the transcriptome of the pvM population, we analyzed the single cell RNA sequencing 140 dataset of the non-neuronal cell population in mouse brain cortex (GSE133283), thus including the $141 \mathrm{CD}^{-}$cells (Fig. 4B, C). We identified macrophages expressing the characteristic macrophage 142 markers Mrc1, Cd68 and Fcgr3 (Fig.S4B). This macrophage cluster was segregated into at least two 143 subtypes; Lyvel $^{+}$and $\mathrm{H}_{2}-\mathrm{Aa}^{+}$(one of the genes encoding MHCII) (Fig.4C). We observed cells with 
144 lower or undetectable Ptprc or Cx3crl expression and fewer cells with lower Spil expression (Fig.4C).

145 However, these cells were not segregated from other cells by unsupervised clustering analysis, 146 confirming their identity as macrophages.

147 Since we ruled out a lymphatic identity, established that the cells expressed macrophage markers and 148 clustered together with the conventional pvM in the single cell sequencing, we name these cells non149 conventional $\mathrm{CD}^{-} 5^{-}$perivascular macrophages (pvM2).

150 PU.1 is considered to be a master regulator for macrophage differentiation. Therefore, we analyzed the 151 Spi1 ${ }^{G F P / G F P}$ mouse model, in which GFP is knocked into the Spil locus (encoding PU.1) rendering this 152 gene inactive. Homozygous mice deficient for Spil lack all macrophages and die around birth ${ }^{8,27}$. In 153 E18.5 Spil $1^{\text {GFP/+ }}$ embryos, we identified Lyve ${ }^{+}$PU. $1^{+} \mathrm{CD} 45^{+}$and $\mathrm{Lyve1}{ }^{+} \mathrm{PU} .1^{-} \mathrm{CD} 45^{-}$pvM (pvM2)

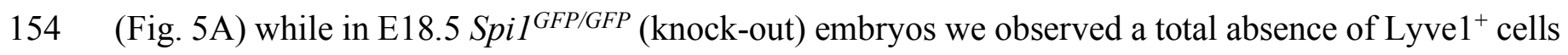
155 in the brain parenchyma (Fig. 5B, positive staining of Lyve $1^{+}$cells in the skull in Supplementary Fig. 156 5). This demonstrates that $\mathrm{Lyve} 1^{+} \mathrm{CD} 45^{-}$depend on PU.1 during their development, but lose expression 157 during life (Supplementary Fig. 3C).

158 We tested a possible hematopoietic stem cell (HSC) - bone-marrow derived hematopoietic origin of 159 this population using the $V a v^{C r e} ; \operatorname{Rosa}_{2} 6^{t d T}$ (hereafter named $\operatorname{Vav}^{t d T}$ ) reporter mouse model. Vav 160 expression starts around embryonic day 8.5 (E8.5) in the hemogenic endothelium of the dorsal aorta in 161 aorta-gonad-mesonephros (AGM) region, which gives rise to the HSC and monocytes ${ }^{36}$. By 162 immunofluorescence staining of $\operatorname{Vav}^{t d T}$ brain sections, we identified a Lyve $1^{+} \mathrm{CD} 45^{+} \mathrm{Vav}^{\mathrm{tineage}}{ }^{+}$and 163 a Lyve $1^{+} \mathrm{CD} 45^{-}$Vav-lineage ${ }^{-}$population (Fig. 5C). Therefore, we concluded that the Lyve $1^{+} \mathrm{CD} 45^{-}$ 164 cells were not derived from HSC or bone-marrow derived monocytes.

165 Phagocytosis by pvM2

166 Besides their role as source for chemokines and growth factors to regulate an immune response, pvM 167 have a phagocytic function, which could be part of a broader role of these cells for tissue homeostasis $168{ }^{19,37}$. In order to address a role of the pvM2 in fluid drainage or macromolecule clearance, we injected 169 10kD Dextran-AlexaFluor647 and Acetylated LDL-AlexaFluor594 in the lateral ventricle of $170 C x 3 \mathrm{crl}^{G F P}$ mouse brains. To exclude recirculation through the blood stream, we analyzed the brain 10 171 minutes after injection ${ }^{3}$. We observed that both $\mathrm{Lyve}^{+} \mathrm{CX} 3 \mathrm{CR} 1^{-}$and $\mathrm{Lyve}^{+} \mathrm{CX} 3 \mathrm{CR} 1^{+}$cells 172 phagocytized the injected dyes near the ventricle, in the superior- (Fig. 6A-C) and in the inferior cortex

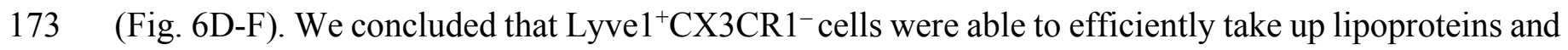
174 glycoproteins in the range of at least 3 to $10 \mathrm{kDa}$ similar as conventional pvM. The close proximity of 175 the pia mater extending towards the third ventricle $(3 \mathrm{~V})$ could explain direct drainage from the 
176 injection-site within the lateral and connected 3V towards the pia mater (Fig. 6G). Very few Lyve1 and

177 Prox1mOrange2 positive nuclei were observed within the pia mater (Fig. 6G).

\section{Involvement in central nervous system diseases}

179 It was shown that in stroke pvM can influence the blood-brain-barrier function ${ }^{38,39}$. In patients with

180 intracerebral hemorrhage and focal cerebral ischemia, a CD163 ${ }^{+}$cell accumulation around brain blood

181 vessels was observed, which also contained myelin ${ }^{40}$. Moreover, it was not clear whether these cells

182 are blood-derived monocytes or pvM since they were not characterized in detail (Holfelder K. et al., 183 2011).

184 To assess the role of the pvM2 in cerebrovascular pathologies, we investigated a photothrombotic 185 model of ischemic stroke. PT was induced on 8 weeks old male mice. In this model, we observed an 186 increase in Lyve1 staining density in whole-mount stained brains (Fig. 7A, method for quantification 187 of staining density in whole mount brain shown in Supplementary Fig. 6A). At day 14 post stroke 188 induction (P14), we noticed a significant increase of Lyve1 staining density $(4.2 \mathrm{x}, \mathrm{p}<0.05)$ within the

189 hippocampus, although no significant difference was observed in the superior cortex. At P30, Lyve1 190 staining density had normalized to control values again (Fig. 7B). We noted an increase in the total 191 Lyve $^{+}$population, including CD $45^{\text {int }}$ and CD45 ${ }^{-}$population by flow cytometry (Fig. 7C). The relative 192 numbers of the CD45 ${ }^{\text {int }}$ and CD45- populations remained similar compared to the control (not shown), 193 but the absolute number of CD45- ${ }^{-} y v e 1^{+} \mathrm{F} 4 / 80^{+}$pvM2 increased in PT (Fig. 7D, E and Supplementary 194 Fig. 6B). 
196 We have carefully examined whether lymphatic endothelial cells exist within the central nervous system. However, thorough analysis of different reporter mouse models did not reveal these cells within the parenchyma and few individual lymphatic endothelial cells were observed only within the pial meninges as described before ${ }^{35}$. Using flow-cytometry, single cell, immunofluorescence and lineage-tracing models, we characterized a novel perivascular cell population and named it nonconventional $\mathrm{CD}^{-}$perivascular macrophages (pvM2). This population did not express several classical macrophage markers but still clustered together with other macrophages in single cell sequencing analysis. It closely resembled conventional pvM, in location and function. Similar as pvM, pvM2 located outside the cul-de-sac of the pia-mater and within the Virchow-Robin space, which is likely to be associated with their functioning in drainage $8,39,41$. Classical brain pvM are derived from the yolk-sac hemogenic endothelium and depend on Csf1R during their maturation ${ }^{8}$. Based on the absence of labeling in the $\operatorname{Vav} 1^{t d T}$ model, we conclude that pvM2 are not bone-marrow derived monocytes. As was shown for pvM in the periphery ${ }^{19}$, pvM2 are also most likely derived from the yolk-sac. Using the Spil reporter (encoding the PU.1 protein) and knock-out model, we demonstrate that, similar to myeloid cells, they require PU.1 for differentiation but not for maintenance. They could still have the Spil mRNA transcripts, but not he protein or GFP reporter, as we observed more cells positive for Spil than for Cx3crl or Ptprc in the single cell sequencing analysis. It was previously noted that some macrophages originated from $\mathrm{CX} 3 \mathrm{CR} 1^{+}$precursors but ceased to express $\mathrm{Cx} 3 \mathrm{cr} 1$, such as alveolar macrophages, Langerhans and Kupffer cells. These $\mathrm{CX} 3 \mathrm{CR}^{-}$cell coincided with $\mathrm{CX} 3 \mathrm{CR} 1^{+}$cells ${ }^{42}$, as we also observed in the brain parenchyma. Why pvM2 lacked Csf1R and thus likely do not require Csf1 for maintenance is yet unclear. Further experiments using other lineage tracing models are required to reveal their exact origin in relation to the differentiation of conventional pvM. It was observed before that non-hematopoietic ectodermal cells gave rise to phagocytosing cells, resembling Langerhans cells, in the skin of zebrafish ${ }^{43}$. However, it was not shown that these cells depend on PU.1 and moreover were located within the skin of the zebrafish. Whether these cells resemble the murine pvM2 is yet unclear.

In adult tissues at steady-state, pvM have important functions related to their perivascular location, such as the regulation of vascular permeability, phagocytosis of blood-transmissible pathogens, antigen presentation or immune regulation ${ }^{37}$. Here, we observed similar phagocytosis of the pvM2 when compared to conventional pvM. Previously, two perivascular monocyte populations were described, being Lyve1 ${ }^{\text {hi }}$ or Lyve ${ }^{\text {lo }}$. MHCII was expressed high on the Lyve1 ${ }^{\text {lo }}$ population while the Lyve ${ }^{\text {hi }}$ population was MHCII low ${ }^{19}$. However, in past studies both Lyve1 ${ }^{\text {hi }}$ or Lyve ${ }^{\text {lo }}$ populations were gated 
only from $\mathrm{CD} 45^{+}$cells. CD45 negative non-conventional pvM, if present, were disregarded. Since the

229 pvM2 did not express CD45, MHCII nor CX3CR1, but were Lyve1 ${ }^{+}$, it is not clear how these cells

230 relate to the previously described perivascular monocytes.

231 Using whole-mount imaging enabled us to establish the pvM contribution specifically within and 232 adjacent to the affected region in ischemic stroke. We established an increase in the total pvM number 233 after stroke, with a concomitant significant increase of the pvM2 population. As the number of the 234 pvM2 increases during stroke, these cells may be involved in the generation of an activated immune 235 status. All previous studies on pvM within the brain analyzed CD45 cells. This study now for the first 236 time presents evidence of a $\mathrm{CD} 45^{-} \mathrm{CX} 3 \mathrm{CR}^{-}$population, named pvM2, which is present within the 237 brain and should be considered in the future to better understand the active role of diverse macrophage 238 populations in brain homeostasis and disease.

240 In conclusion, here we demonstrate the existence and function of non-conventional CD45- pvM2, 241 lacking classical macrophage makers such as CD45, CX3CR1, PU.1, CD206, CD163 and CD11b. 242 These cells are consistently observed within the brain and are likely involved in drainage also due to 243 their blood vessel proximity and involved in an activated immune state such as during ischemic stroke. 244 Although these novel pvM2 increase in number during stroke, their specific role during disease remains 245 unclear. Presently, there are no unique markers available to target these cells specifically, but a newly 246 developed marker should allow a targeted approach to study their specific role in brain physiology in 247 the future. 


\section{Mice}

$250 \mathrm{C} 57 \mathrm{BL} / 6 \mathrm{~J}$ and Vav1-Cre ${ }^{23}$ mice were obtained from Charles River [France]. Prox-CreERT2 $2^{+/-24}$ and

251 Wnt1-Cre ${ }^{25}$ mice were kindly provided by Dr. Bajénoff (CIML, Marseille, France). Cx3crl $1^{G F P}$ mice $252{ }^{26}$ were kindly provided by Dr. Lelouard (CIML, Marseille, France). Spi1 ${ }^{\text {GFP }}$ (PU.1) ${ }^{27}$, Csf1R-Cre ${ }^{28}$ 253 and Prox $1^{\text {mOrange } 2} 29$ were maintained at the CIML (Marseille, France).

254 Vav1-Cre, Prox-CreERT2 ${ }^{+-}$, Wnt1-Cre and Cx3crl-Cre lines were crossed to homozygosity for the 255 tdTomato reporter using Rosa $26^{\text {tdT }}$ mice ${ }^{30} .5 \mathrm{mg}$ of Tamoxifen was injected IP to a Prox-CreERT2 ${ }^{+/-}$ 256. Rosa26 $6^{\mathrm{tdT}}$ adult 2 weeks before the isolation of the brain. All experiments were reviewed and approved 257 by the local ethics committee of Aix-Marseille University and the Ministère de l'Enseignement 258 Supérieur, de la Recherche et de l'Innovation.

$260 \quad$ Antibodies Table 1

\section{Cell preparation and flow cytometry}

$262 C x 3 c r 1^{G F P}$ mouse brains from male mice were collected after perfusion with ice cold PBS-Heparin 263 (after removal of the dura). Brains were cut sagitally in 6-8 pieces, then digested with the Adult Brain 264 Dissociation Kit (Miltenyi, 130-107-677) at $37^{\circ} \mathrm{C}$ for $30 \mathrm{~min}$ on the gentleMACS dissociator 265 (Miltenyi). Brain cell suspensions were filtered over $70 \mu \mathrm{m}$ strainers and filters were washed with $10 \mathrm{~mL}$ 266 HBSS $2 \%$ FBS. After centrifugation $\left(5 \mathrm{~min}, 400 \mathrm{~g}, 4^{\circ} \mathrm{C}\right)$, debris were removed using a $40 \%$ Percoll 267 (Sigma-Aldrich, GE17-0891-02) solution in PBS. Cells were centrifuged (30min, 500g, $4^{\circ} \mathrm{C}$ ) and 268 supernatant was discarded. Red blood cell lysis was performed for 10 min at $4^{\circ} \mathrm{C}$ using the Miltenyi 269 RBC lysis buffer from the kit. Lysis was stopped with 9mL HBSS 2\%FBS and cells were centrifuged $270 \quad\left(5 \mathrm{~min}, 400 \mathrm{~g}, 4^{\circ} \mathrm{C}\right)$. Cells were then blocked (15\% normal mouse serum (Jackson Immunoresearch 015$271000-120$ ) in FACS buffer (HBSS 2\% FBS) for 15 min and subsequently stained for CD45-BUV395 272 (BD biosciences 564279), Lyve1-eFluor660 (eBioscience 50-0443-82) and F4/80-BV421 (Biolegend 273 123131) diluted in FACS buffer for 30min on ice. The staining of dead cells with Fixable NIR was 274 performed for another 30 min after the antibody staining in protein free HBSS right after washing the 275 cells. Cells were subsequently washed and resuspended in FACS buffer. Samples were acquired on the 276 LSRFortessa X-20 cytometer (BD Biosciences). Data analyses were done using FlowJo software 277 (version 10, FlowJo, LLC).

\section{Cell sorting}


279 Cells were prepared and stained with CD45, and Lyve1 antibodies and Fixable NIR as describe above.

280 Live Lyve $1^{+}$CD45-, live Lyve $1^{+}$CD $45^{\text {int }}$ and live Lyve $1^{+}$CD $45^{\text {high }}$ cells were sort-purified separately 281 using the BD FACS ARIA III SORP sorter.

282 RNA isolation and gene expression analysis

283 After spinning down ( $7 \mathrm{~min}, 300 \mathrm{~g}, 4^{\circ} \mathrm{C}$ ), cells were resuspended in $1 \mathrm{~mL}$ TRIzol reagent (Sigma T9424).

284 The suspension was transferred to a phase lock gel heavy tube to facilitate the extraction and $200 \mu \mathrm{L}$ of chloroform was added. The samples were shaken vigorously and incubated for 3 min at room temperature (RT) prior to centrifugation $\left(10 \mathrm{~min}, 12000 \mathrm{~g}, 4^{\circ} \mathrm{C}\right)$. The clear aqueous phase was transferred to a new tube and $2 \mu \mathrm{L}$ of GlycoBlue Coprecipitant (Invitrogen AM9515) was added to help with the isolation prior to adding $500 \mu \mathrm{L}$ of isopropanol. The samples were incubated for $10 \mathrm{~min}$ at RT and subsequently centrifuged (30min, $12000 \mathrm{~g}, 4^{\circ} \mathrm{C}$ ). The supernatant was discarded and RNA was washed with $800 \mu \mathrm{L}$ EtOH $70 \%$. The samples were centrifuged $\left(5 \mathrm{~min}, 12000 \mathrm{~g}, 4^{\circ} \mathrm{C}\right.$ ) and supernatants were discarded. The RNA was air-dried for $15 \mathrm{~min}$ prior to resuspension in $11.5 \mu \mathrm{L}$ RNase free water.

292 The tubes were subsequently heated at $65^{\circ} \mathrm{C}$ for $10 \mathrm{~min}$ and then put on ice. RNA quantity and quality were assessed by nanodrop. For each sample, 200ng of RNA was reverse transcribed using the RevertAid Reverse Transcription kit (Thermo Scientific K1691) and adding Oligo (dT) ${ }_{12-18}$ primer (Invitrogen, 12418012). The level of expression of CD45 (Forward CCCCGGGATGAGACAGTTG; Reverse AAAGCCCGAGTGCCTTCCT) gene was assessed by qPCR using Sybr Green (Takara, TAKRR420W) and Hprt was used as reference gene (Forward GGCCAGACTTTGTTGGATTT; Reverse CAGATTCAACTTGCGCTCAT). Data were analyzed using the delta-delta Ct method and compared to the $\mathrm{CD} 45^{-}$fraction.

Single cell RNA sequencing data of non-neuronal cell populations in mouse brain cortex was adapted from GSE133283 (unpublished). Mrc1-expressing perivascular macrophage population was isolated and reanalysed using Seurat (version 3.2.1) R package. Variable genes were found with parameters of selection.method $=$ vst and nfeatures $=2,000$, trimmed for the genes related to cell cycle $(\mathrm{GO}: 0007049)$ and then used for principal component analysis (RunPCA, npcs=30). Statistically significant principal components were determined by JackStraw method and the first 5 principle components were used for non-linear dimensional reduction (RunTSNE) and clustering analysis (FindNeighbors) with resolution $=0.1$. FeaturePlot function was used to visualize the specific gene expression on TSNE plot. Blue represents the relative expression level of each gene.

\section{Whole-mount staining and lightsheet imaging}

310 Both male and female mice were perfused with PBS-Heparin 5U/ml (Sigma) and subsequently 311 overnight $(\mathrm{ON})$ immersion fixed in paraformaldehyde 4\% (PFA-Electron Microscopy Science, ref 
312 15714) in PBS. The iDISCO protocol was used for immuno-staining ${ }^{31}$, prior to which brains were

313 dehydrated in increasing methanol (MetOH) concentrations diluted in PBS (20, 40, 60, 80 and twice

$314100 \%$ ) for 30 minutes for each concentration at RT. Subsequently specimens were incubated ON in a

315 dichloromethane (DCM, Sigma 270997)-MetOH mixture (2 vol DCM:1 vol MetOH) at RT. After two

31610 minutes incubations in absolute $\mathrm{MetOH}$, brains were bleached in $5 \% \mathrm{H}_{2} \mathrm{O}_{2}$ in $\mathrm{MetOH} \mathrm{ON}$ at $4{ }^{\circ} \mathrm{C}$

317 and subsequently rehydrated by a decreasing MetOH series (80, 60, 40, 20\% in water), followed by

318 PBS and two washes in PBS-Triton X100 (Tx) $0.2 \%$ for 1 hour each. Bleached brains were

319 permeabilized for 2.5 days at $37^{\circ} \mathrm{C}(0.4 \% \mathrm{Tx}, 20 \% \mathrm{DMSO}, 2.3 \%$ Glycine in PBS) and subsequently

320 blocked with [PTwH (PBS, Tween20, Heparin), 10\% DMSO, 6\% serum] for 4.5 days at $37^{\circ} \mathrm{C}$. Whole-

321 mount stainings were performed by incubation with primary antibodies for 5 days at $37^{\circ} \mathrm{C}$ and

322 subsequently with Alexa-dye coupled secondary antibodies diluted in PTwH containing 3\% serum for

3235 days at $37^{\circ} \mathrm{C}$. Following each staining step, samples were extensively washed in PTwH (10min,

$32415 \mathrm{mn}, 30 \mathrm{~min}, 1 \mathrm{~h}, 2 \mathrm{~h}$, and $\mathrm{ON}$ at RT). Finally, the samples were again dehydrated by increasing $\mathrm{MetOH}$

325 concentrations diluted in water $(20,40,60,80,2 \times 100 \%$ and $\mathrm{ON}$ in absolute MetOH), for 1 hour each

326 step at RT and cleared in a MetOH/BABB mix (1:1) [BABB (benzyl alcohol and benzyl benzoate =

327 1:2) (Sigma 305197 and Fisher Scientific 10654752)] for 8 hours at RT and finally placed in BABB

$328 \mathrm{ON}$ at RT to complete clearing. All incubations were done under mild agitation. After clearing, brains

329 were imaged using a LaVision Ultramicroscope II (LaVision BioTec, Bielefeld, Germany). Stacks

330 were captured with a step size of $5 \mu \mathrm{m}$ at $2.5 \mathrm{X}$ magnification using an optic zoom with a NA=0.144.

$3313 \mathrm{D}$ reconstruction, cell counting and analysis of the sample image stacks were performed using

332 IMARIS software (Version 9.1.0, Bitplane). For Lyve1 quantification, an area of interest was selected

333 (Superior cortex, Hippocampus) using the program's surface function and then a new channel

334 corresponding to the fluorescence to quantify was created in this region. Using this workflow, a

335 quantification of volume (in $\mu \mathrm{m}^{3}$ ) of Lyve1 stained material is possible, which was further adapted for

336 volumetric quantifications (Supplementary Fig. 6).

337 Vibratome section immunofluorescence staining and confocal imaging

338 Animals were perfused with PBS/heparin, brains dissected and fixed $\mathrm{ON}$ in $4 \% \mathrm{PFA}$ in $\mathrm{PBS}$ at $4^{\circ} \mathrm{C}$

339 and subsequently embedded in 1\% low melting agarose for generation of $100 \mu \mathrm{m}$ vibratome slices

340 (Leica, VT1000S). Sections were blocked in EBT buffer (EBSS, 0.05\% Tx, 1\% BSA) containing 10\%

341 serum for $2 \mathrm{~h}$ at RT under agitation. Immunostainings were performed by incubation in primary

342 antibodies for $48 \mathrm{~h}$ at $4^{\circ} \mathrm{C}$ in EBT, 3\% sera and subsequently with Alexa-fluorochrome coupled

343 secondary antibodies diluted in EBT, 3\% sera for $24 \mathrm{~h}$ at $4{ }^{\circ} \mathrm{C}$. Following each staining step, samples

344 were washed several times in PBS-Tx (0.05\% Tx in PBS) and in PBS. Sections were finally cleared in 
345 Histodenz (Sigma D2158) medium for 48h at RT and subsequently mounted in Histodenz medium.

346 Confocal images were acquired at RT on a confocal microscope (LSM880, Zeiss, Germany), with a

347 20x/0.4 Plan-Apochromat objective and using laser lines at 405, 488, 561, and 633nm for the excitation

348 of AlexaFluor405/GFP/AlexaFluor555/AlexaFluor647 respectively. Fluorescence was recorded in

349 individual channels acquired in a sequential mode using a highly sensitive 32-channel GaAsP detector.

350 Channels were respectively detected using these detection bands: A405 (410-470nm), A488 (490-

$351540 \mathrm{~nm})$, A555 (565-640nm), A647 (640-690nm). The pinhole was set to 1 airy unit. Z stack were

352 acquired with an optical thickness defined for each image in figure legends, satisfying the Nyquist

353 resolution criterion. Image processing (contrast enhancement, scale bars, etc.) was done with ImageJ

354 (National Institutes of Health) without actions modifying image integrity.

\section{Infusion of tracers into lateral ventricle}

356 Both female and male mice were anesthetized (150mg/ $\mathrm{kg}$ Ketamine and $10 \mathrm{mg} / \mathrm{kg}$ Xylazine) and fixed 357 in a stereotaxic frame. The skull was thinned with a dental drill at a location of $0.95 \mathrm{~mm}$ lateral and $3580.22 \mathrm{~mm}$ caudal from the bregma. A 30G needle with a silica fiber tip (Phymed) was inserted into the 359 right lateral ventricle $2.35 \mathrm{~mm}$ ventral to the skull surface as previously described ${ }^{3}$. Injection of 2.5 $360 \mu \mathrm{L}$ acetylated LDL-Alexa594 (ThermoFisher) or Dextran-Alexa647 10kDa (ThermoFisher) tracer was 361 done at a speed of $0.5 \mu \mathrm{L} / \mathrm{min}$ using a high precision syringe pump. The needle was left in place for 5 362 minutes and slowly retracted confirming lack of detectable backflow. To avoid recirculation through 363 the blood stream into the brain, mice were sacrificed 10 min after injection ${ }^{3}$. The mice were perfused 364 with PBS and 4\%PFA/PBS, brains isolated and fixed ON in 4\% PFA/PBS at $4{ }^{\circ} \mathrm{C}$, subsequently washed 365 and then stored at $4^{\circ} \mathrm{C}$ in PBS until further analysis.

366 Photothrombotic (PT) induced stroke

367 Stroke using the PT model was induced in male $\mathrm{C} 57 \mathrm{BL} / 6 \mathrm{~J}$ and $\mathrm{Cx} 3 \mathrm{cr} \mathrm{1}^{\mathrm{GFP}}$ mice. Mice were 368 anesthetized by intraperitoneal Ketamine/Xylazine injection as before and the eyes of the mice were 369 covered with Ocry-gel to protect them from light and dehydration. The skin on the skull was incised 370 from the eyes to the neck and retracted to the edges of the skull. After retro-orbital injection of $100 \mu \mathrm{L}$ 371 Rose Bengal (Sigma, 330000) a Leica, KL 1600LED cold light source was placed in contact with the 372 skull and the illuminated region was precisely adjusted (using a stereotaxic instrument) to $2.5 \mathrm{~mm}$ 373 caudal of the Bregma and $2.0 \mathrm{~mm}$ to the Lambda. After illuminating a $1 \mathrm{~mm}$ diameter area at power 3 374 for 15 minutes, the skin was put back into place, stitched and the animals returned to their home cages.

375 Food was provided in a plate and Buprenorphine $(0.4 \mathrm{mg} / \mathrm{ml})$ was added to the drinking water. Mice 376 were sacrificed between 14- and 30-days post-induction and brains were analyzed by 377 immunofluorescence and flow cytometry. 
379 Graphs, average values and standard deviation (SD) shown in all figures were calculated using Prism

380 (version 8.3.0 GraphPad software) software. Unpaired T-Test and One-way Anova test were used to 381 determine significance. The number of individual experiments can be found in the legends of all the 382 figures. Photoshop software (CC2015, Adobe) was used to generate figures.

\section{REFERENCES}

385 1. Aspelund, A. et al. A dural lymphatic vascular system that drains brain interstitial fluid and 386 macromolecules. J. Exp. Med. 212, jem.20142290- (2015).

387 2. Ahn, J. H. et al. Meningeal lymphatic vessels at the skull base drain cerebrospinal fluid.

388 Nature (2019). doi:10.1038/s41586-019-1419-5

389 3. Ma, Q., Ineichen, B. V., Detmar, M. \& Proulx, S. T. Outflow of cerebrospinal fluid is predominantly through lymphatic vessels and is reduced in aged mice. Nat. Commun. 8, 1434

392 4. van Lessen, M. et al. Intracellular uptake of macromolecules by brain lymphatic endothelial cells during zebrafish embryonic development. Elife 6, (2017).

5. Absinta, M. et al. Human and nonhuman primate meninges harbor lymphatic vessels that can be visualized noninvasively by MRI. Elife 6, e29738 (2017).

6. Louveau, A. et al. Structural and functional features of central nervous system lymphatic

8. Goldmann, T. et al. Origin, fate and dynamics of macrophages at central nervous system interfaces. Nat. Immunol. 17, 797-805 (2016).

9. Zhang, E. T., Inman, C. B. \& Weller, R. O. Interrelationships of the pia mater and the vessels. Nature (2015). doi:10.1038/nature14432

7. Prinz, M., Erny, D. \& Hagemeyer, N. Ontogeny and homeostasis of CNS myeloid cells. Nat. Immunol. 18, 385-392 (2017). perivascular (Virchow-Robin) spaces in the human cerebrum. J. Anat. 170, 111-123 (1990).

10. Mato, M., Ookawara, S. \& Kurihara, K. Uptake of exogenous substances and marked infoldings of the fluorescent granular pericyte in cerebral fine vessels. Am. J. Anat. 157, 329332 (1980).

11. Mato, M. et al. Involvement of specific macrophage-lineage cells surrounding arterioles in barrier and scavenger function in brain cortex. Proc. Natl. Acad. Sci. 93, 3269 LP - 3274 (1996).

12. Bechmann, I. et al. Immune surveillance of mouse brain perivascular spaces by blood-borne 
macrophages. Eur. J. Neurosci. 14, 1651-1658 (2001).

412 13. Fabriek, B. O. et al. CD163-positive perivascular macrophages in the human CNS express molecules for antigen recognition and presentation. Glia 51, 297-305 (2005).

414 14. Faraco, G. et al. Perivascular macrophages mediate the neurovascular and cognitive

415 dysfunction associated with hypertension. J. Clin. Invest. 126, 4674-4689 (2016).

416 15. Hawkes, C. A. \& McLaurin, J. Selective targeting of perivascular macrophages for clearance 417 of $\beta$-amyloid in cerebral amyloid angiopathy. Proc. Natl. Acad. Sci. 106, 1261 LP - 1266 418 (2009).

419 16. Thanopoulou, K., Fragkouli, A., Stylianopoulou, F. \& Georgopoulos, S. Scavenger receptor 420

21. Utz, S. G. et al. Early Fate Defines Microglia and Non-parenchymal Brain Macrophage Development. Cell 181, 557-573.e18 (2020).

22. Ydens, E. et al. Profiling peripheral nerve macrophages reveals two macrophage subsets with distinct localization, transcriptome and response to injury. Nat. Neurosci. 23, 676-689 (2020).

23. Joseph, C. et al. Deciphering Hematopoietic Stem Cells in Their Niches: A Critical Appraisal

24. Bazigou, E. et al. Genes regulating lymphangiogenesis control venous valve formation and maintenance in mice. J. Clin. Invest. 121, 2984-92 (2011). of Genetic Models, Lineage Tracing, and Imaging Strategies. Cell Stem Cell 13, 520-533 (2013).

25. Brault, V. et al. Inactivation of the $(\beta)$-catenin gene by Wnt1-Cre-mediated deletion results in dramatic brain malformation and failure of craniofacial development. Development 128, 1253 LP - 1264 (2001).

26. Jung, S. et al. Analysis of Fractalkine Receptor CX3CR1 Function by Targeted Deletion and Green Fluorescent Protein Reporter Gene Insertion. Mol. Cell. Biol. 20, 4106-4114 (2000). 
27. Back, J., Dierich, A., Bronn, C., Kastner, P. \& Chan, S. PU.1 determines the self-renewal capacity of erythroid progenitor cells. Blood 103, 3615-3623 (2004).

28. Li, J., Chen, K., Zhu, L. \& Pollard, J. W. Conditional deletion of the colony stimulating factor1 receptor (c-fms proto-oncogene) in mice. genesis 44, 328-335 (2006).

29. Hägerling, R., Pollmann, C., Kremer, L., Andresen, V. \& Kiefer, F. Intravital two-photon microscopy of lymphatic vessel development and function using a transgenic Prox1 promoterdirected mOrange2 reporter mouse. Biochem. Soc. Trans. 39, 1674-81 (2011).

30. Madisen, L. et al. A robust and high-throughput Cre reporting and characterization system for the whole mouse brain. Nat. Neurosci. 13, 133-140 (2010).

31. Renier, N. et al. iDISCO: A Simple, Rapid Method to Immunolabel Large Tissue Samples for Volume Imaging. Cell 159, 896-910 (2014).

32. Engelhardt, B., Vajkoczy, P. \& Weller, R. O. The movers and shapers in immune privilege of the CNS. Nat. Immunol. (2017). doi:10.1038/ni.3666

34. Hägerling, R. et al. A novel multistep mechanism for initial lymphangiogenesis in mouse embryos based on ultramicroscopy. EMBO J. 32, 629-44 (2013).

35. Shibata-Germanos, S. et al. Structural and functional conservation of non-lumenized lymphatic endothelial cells in the mammalian leptomeninges. Acta Neuropathol. 139, 383-401 (2019).

38. Koizumi, T., Kerkhofs, D., Mizuno, T., Steinbusch, H. W. M. \& Foulquier, S. Vessel-

36. Bustelo, X. R., Suen, K. L., Leftheris, K., Meyers, C. A. \& Barbacid, M. Vav cooperates with Ras to transform rodent fibroblasts but is not a Ras GDP/GTP exchange factor. Oncogene $\mathbf{9}$, 2405-2413 (1994).

37. Lapenna, A., De Palma, M. \& Lewis, C. E. Perivascular macrophages in health and disease. Nat. Rev. Immunol. (2018). doi:10.1038/s41577-018-0056-9 Associated Immune Cells in Cerebrovascular Diseases: From Perivascular Macrophages to Vessel-Associated Microglia. Front. Neurosci. 13, 1291 (2019).

39. Yang, T., Guo, R. \& Zhang, F. Brain perivascular macrophages: Recent advances and implications in health and diseases. CNS Neurosci. Ther. 25, 1318-1328 (2019).

40. Kösel, S. et al. Long-lasting perivascular accumulation of major histocompatibility complex class II-positive lipophages in the spinal cord of stroke patients: possible relevance for the immune privilege of the brain. Acta Neuropathol. 94, 532-538 (1997). 
477 41. Prinz, M. \& Priller, J. The role of peripheral immune cells in the CNS in steady state and 478 disease. Nat Neurosci 20, 136-144 (2017).

479 42. Yona, S. et al. Fate Mapping Reveals Origins and Dynamics of Monocytes and Tissue $480 \quad$ Macrophages under Homeostasis. Immunity 38, 79-91 (2013).

481 43. Lin, X. et al. An Ectoderm-Derived Myeloid-like Cell Population Functions as Antigen 482 Transporters for Langerhans Cells in Zebrafish Epidermis. Dev. Cell 49, 605-617.e5 (2019). 


\section{Competing Interests}

484 The authors declare that the research was conducted in the absence of any commercial or financial 485 relationships that could be construed as a potential conflict of interest.

486 Author Contributions

487 CS, ML, HWJ, MS, LF, AT, performed experiments. SAvdP conceived and supervised the study. CS, 488 ML, HWJ, MS, and SAvdP analyzed data. CS, ML, HWJ, MS, AT, MF, SS, MHS, RS, RHA, SSM, 489 FK and SAvdP contributed to discussion and wrote, illustrated, reviewed, and edited the manuscript. 490 SAvdP acquired funding. All authors approved the submitted version of the manuscript.

491 Acknowledgments

492 The work was supported by the FRM Amorçage jeunes équipes (AJE20150633331), ANR ACHN 493 (ANR-16-ACHN-0011), ANR PRCI (ANR-17-CE13-0029-01), A*midex Chaire d'excellence to 494 SAvdP, DFG (FOR2325 to S.S.-M) and institutional grants to the CIML from INSERM, CNRS and 495 Aix-Marseille University.

496 We acknowledge the PICSL imaging facility of the CIML (ImagImm), member of the national 497 infrastructure France-BioImaging supported by the French National Research Agency (ANR-10498 INBS-04), notably Mathieu Fallet and Sebastian Mailfert. Lionel Chasson of the histology platform 499 for the relentless cutting. We thank the animal facility, notably Toufik Guelmami and Michel Pontier.

500 The flow cytometry core facility, notably Marc Barad, Sylvain Bigot and Laurence Borge. We thank

501 Hugues Lelouard and Marc Bajénoff for providing us with mouse models and Lydia Sorokin for the 502 anti- Laminin $\gamma 1$ antibody.

503 Data Availability Statement

504 All datasets generated for this study are included in the article/Supplementary Material. Single cell 505 sequencing data is available under GSE133283. 
507 Figure 1: Identification of a Lyve1 ${ }^{+}$CX3CR1- $^{-}$population in the mouse brain. Confocal microscopy of brain sections stained with $\alpha$ Lyve1 showing the different Lyve ${ }^{+}$cell morphologies in the superior cortex $(17 \mu \mathrm{m}$ maximum intensity projection) (A), in the inferior cortex $(27 \mu \mathrm{m}$ maximum intensity

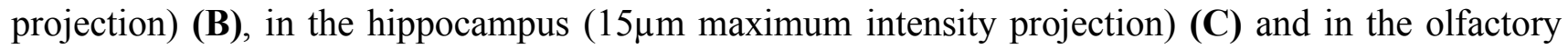
bulb $(61 \mu \mathrm{m}$ maximum intensity projection) (D) of an adult mouse brain. (E-I) Immunofluorescence microscopy on sections of $\mathrm{Cx} 3 \mathrm{cr} 1^{G F P}$ and $\mathrm{Spil}^{G F P}$ (encoding PU.1) in the superior cortex of the mouse brain. (E) Staining of $C x 3 c r 1^{G F P}$ sections for Lyve1 (red) and F4/80 (50 $\mu \mathrm{m}$ maximum intensity projection), (F) Ibal ( $29 \mu \mathrm{m}$ maximum intensity projection),

(G) CD45 (46 $\mu \mathrm{m}$ maximum intensity

(I) Staining of $\operatorname{Spil}^{G F P}$ (PU.1 - green)

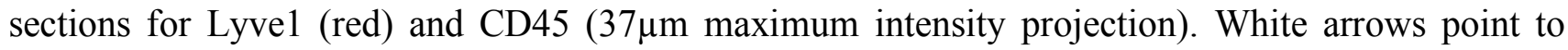
conventional Lyve $1^{+} \mathrm{CX} 3 \mathrm{CR} 1^{+}$pvM cells, which expressed $\mathrm{F} 4 / 80^{+}, \mathrm{Iba1}^{+}, \mathrm{CD} 45^{+}$and $\mathrm{Csf} 1 \mathrm{R}^{+}$. The red arrows indicate $\mathrm{Lyve} 1^{+} \mathrm{CX} 3 \mathrm{CR} 1^{-}$cells, which are $\mathrm{F} 4 / 80^{+}, \mathrm{Iba}^{+}, \mathrm{CD} 45^{-}$and $\mathrm{Csf1R}{ }^{-} \mathrm{PU} .1^{-}$.

Figure 2: Perivascular Lyve1 ${ }^{+}$cell location. (A) Maximal projection $(200 \mu \mathrm{m}$ maximum intensity projection) of a lightsheet microscope acquisition on a cleared mouse brain labeled for SMA (green), CD31 (red) and Lyve1 (blue). (B) Confocal microscopy of brain sections stained for Podoplanin (green), Lyve1 (red) and CD31 (blue), inserts (C) and (D) zoom in on Lyve1 ${ }^{+}$cells lining the blood vessels and placed outside of the «cul de sac of the pia mater » defined by the podoplanin labeling. (E) Confocal microscopy of a superior cortex section stained for CD31 (green), LamininY1 (red) and Lyve1 (blue) with insert (F) showing a higher magnification showing the Lyve $^{+}$cells in the perivascular space. (G) Lyve ${ }^{+}$cell characterization by immunofluorescence on adult brain sections for Lyve1 (green), VEGFR3 (red) and CD31 (blue) staining. The arrowhead points at a blood vessel and the white arrow shows a Lyve $1^{+}$cell negative for VEGFR3 and CD31. (H) high magnification of the Lyve1+ cell in (G). (I and J) Lyve ${ }^{+}$cells (green) did not express Prox1 (red) in the superior cortex $(39 \mu \mathrm{m}$ maximum intensity projection) nor in the inferior cortex $(50 \mu \mathrm{m}$ maximum intensity projection) ( $K$ and $L)$.

534 Figure 3: Confirmation of the $\operatorname{Lyve}^{+} \mathbf{C X} 3 \mathbf{C R} 1^{-}$population by flow cytometry. Flow cytometric analysis of $C x 3 \mathrm{crl}^{\text {GFP }}$ brain parenchyma, pre-gated on living, single and Lyve $1^{+}$cells. Microglia cells were excluded since these are Lyve1 ${ }^{-}$. Mouse brain were analyzed at (A) P2 (n=4), (B) P7 (n=4), (C) 
showing subsequent CX3CR1, F4/80 expression for the cells of the CD45 ${ }^{\text {Int }}$ and $C D 45^{-}$gates. (G) Total number of Lyve $1^{+} \mathrm{CD} 45^{-} \mathrm{CX} 3 \mathrm{CR} 1^{-} \mathrm{F} 4 / 80^{+}$cells at the different stages.

541 Figure 4: Transcriptional profiling of the pvM subsets. A) Quantification by RT-QPCR of the 542 relative expression of Ptprc (encoding CD45) within the CD45 cell subpopulations (CD45- for CD45 negative, CD45 I for intermediate and CD45 H for high expressing CD45) isolated from C57BL/6J brain parenchyma ( 3 sorts with $n=5$ mice per sort), (B) Single cell RNA sequencing of non-neuronal population, focused on the macrophage subset, revealed two main populations. (D) A segregation occurred between a Lyve ${ }^{+}$vs. MHCII (e.g. H2-Aa) macrophage subsets, visualized in the individual gene expressions plotted within the tSNE plots. Ptprc and Cx3 crl transcripts were present in few cells while Spil (encoding PU.1) transcripts were present in more Lyve1 and MHCII cells.

Figure 5: Origin of Lyve1 $^{+}$cells. (A) Immunofluorescence on sections of the head of a E18.5 Spi1 $1^{\text {GFP/+ }}$ embryo, labeled for Lyve1 (red) and CD45 (60 $\mu \mathrm{m}$ maximum intensity projection) shows the presence of the conventional (white arrows) and the non-conventional pvM population (red arrows) at this stage. (B) No GFP nor Lyve1 fluorescence was observed in the Spi $1^{\text {GFP/GFP E1 }} .5$ superior cortex

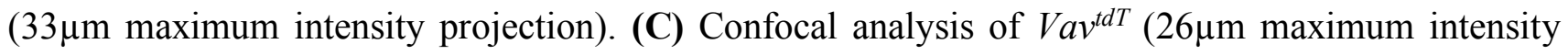
projection) brain sections stained for Lyve1 (green) and CD45 (white). White arrows indicate the conventional pvM population, which is tdT positive (red) and the red arrows the $\mathrm{CD} 45^{-} \mathrm{CX} 3 \mathrm{CR} 1^{-}$ population which is tdT negative.

Figure 6: pvM2 share phagocytic functioning with conventional pvM. (A-C) $C x 3 c r 1^{G F P}$ mouse brains were injected intra-ventricular with Dextran-Alexa647 (37 $\mu \mathrm{m}$ maximum intensity projection) and (D-F) Acetylated-LDL-Alexa594 (42 $\mu \mathrm{m}$ maximum intensity projection). Ten minutes after injection, mice were sacrificed and brains dissected. Confocal analysis on brain sections shows Lyve1 (white), Dyes (red) and CX3CR1 (green). The pvMs phagocytosed the dyes (red arrows), as well as did conventional pvM (white arrows) (B, C, E and F). (G) Tiled confocal acquisition of a Prox $1^{\text {mOrange } 2}$ brain section. Lyve ${ }^{+}$cells in green lined blood vessels stained for CD31 in blue. Lyve $1^{+}$cells in green penetrated the brain to the hippocampal fissure (hif) and all the way towards the third ventricle (3V).

Figure 7: pvM2 increase in numbers after induced ischemic stroke. (A) Maximal intensity projections $(2660 \mu \mathrm{m})$ of lightsheet acquisitions on cleared brains, stained for Lyve1 (red) and CD45 (white), control and after stroke (P14=14 days after induction of ischemic stroke). (B) Quantification 
571 of Lyve1 in the area containing the whole stroke lesion within the superior cortex situated just above 572 the hippocampus, imaged by lightsheet microscopy. (C) Representative flow cytometry plots from $573 \mathrm{Cx} 3 \mathrm{cr}^{G F P}$ brain parenchyma 14 days after induced stroke (P14), pre-gated on living, single and Lyve ${ }^{+}$ 574 cells. Microglia cells were excluded since they are Lyve $1^{-}$. CX3CR1 vs F/80 expression is shown for 575 the $\mathrm{CD} 45^{-}$population. Average percentages for the different subpopulations are shown in the plots 576 ( $\mathrm{n}=4)$. (D) Graph representing the total $\mathrm{Lyve}^{+}$cell number showing an increase of this population at 577 P14 after stroke by flow cytometry. (E) Total pvM2 (Lyve ${ }^{+} \mathrm{CD} 45^{-} \mathrm{CX} 3 \mathrm{CR} 1^{-} \mathrm{F} 4 / 80^{+}$) numbers in the 578 control vs. PT brains at P14. 
581 Table 1: Ab Resources Table for Imaging (confocal and lightsheet microscopy)

582 Table 2: pvM vs pvM2 markers

Supplementary Figure 1: pvM2 characterization. (A) Lightsheet imaging of a cleared adult C57BL/6J mouse brain immunolabeled with $\alpha$ Lyve1 using iDisco+ protocol $(5550 \mu \mathrm{m}$ maximum intensity projection), related to video 1. (B-C) Immunofluorescence microscopy on brain parenchyma sections of (B) Spil ${ }^{G F P}$ and (C) $C x 3 c r 1^{G F P}$ in the superior cortex, using an anti-GFP to enhance the GFP signal (green) with Lyve1 staining labeled in red. (D-G) Brain sections are labeled for Lyve1

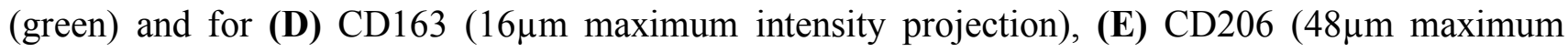

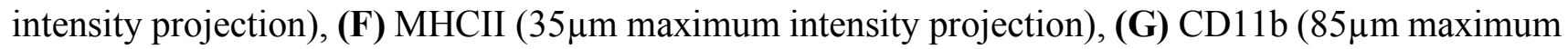
intensity projection) (red). White arrows show the conventional pvM population and red arrows show the non-conventional pvM population.

Supplementary Figure 2: Lyve1 $^{+}$cells are neither lymphatic endothelial cells (LEC), nor astrocytes, nor fibroblasts. (A) $1080 \mu \mathrm{m}$ maximum intensity projection of lightsheet microscope acquisition of the superior cortex illustrating the «cul de sac of the pia mater» defined by the podoplanin labeling, structure emanating from the pia mater (CD31 in green, Lyve1 in red and Podoplanin in blue). (C) Confocal analysis of Prox-CreERT2 ${ }^{+/}$; RosatdTomato brain sections stained for Lyve1 (green) confirmed that Lyve $^{+}$cells do not express tdTomato (red) (white arrows) $(88 \mu \mathrm{m}$ maximum intensity projection). (D) LEC identification $\left(\mathrm{Lyve}^{+} \mathrm{Prox}^{+}\right.$) in the pia mater in the superior cortex by Prox1 antibody staining (red) on C57BL/6J mouse brain in combination with Lyve1 (green). Prox $1^{+}$neuron cellular bodies are observed in the parenchyma ( $13 \mu \mathrm{m}$ maximum intensity projection). (E) Astrocyte identity was excluded by AQP4 and GFAP (red) staining ( $24 \mu \mathrm{m}$ maximum intensity projection for the superior cortex and $17 \mu \mathrm{m}$ maximum intensity projection for the inferior cortex), (F) fibroblast identity was excluded by ER-TR7 staining $(50 \mu \mathrm{m}$ maximum intensity projection for the superior cortex and $8 \mu \mathrm{m}$ maximum intensity projection for the inferior cortex) and (G) PDGFR $\beta$ (red) staining. (H) Confocal analysis of Wnt-Cre; Rosa $26^{\text {tdTomato }}(37 \mu \mathrm{m}$ maximum intensity projection) brain negative $\mathrm{CD}^{4} 5^{-} \mathrm{CX} 3 \mathrm{CR} 1^{-}$perivascular cells. 
611 Supplementary Figure 3: Confirmation of the Lyve1 $^{+} \mathrm{CX}_{3 \mathrm{CR}}{ }^{-}$population by flow cytometry

612 (A) Flow cytometry gating strategy. (B) CD45 $5^{\mathrm{High}}$ cells from $\mathrm{Cx} 3 \mathrm{cr} 1^{G F P}$ brain parenchyma, pre-gated 613 on living, single and Lyve ${ }^{+}$cells. Microglia cells were excluded as Lyve1- ${ }^{-}$Mouse brain analysis at 614 different ages P2 $(\mathrm{n}=4), \mathrm{P} 7(\mathrm{n}=4), \mathrm{P} 14(\mathrm{n}=4), \mathrm{P} 21(\mathrm{n}=4)$, Adult $(8-12$ weeks $)(\mathrm{n}=4)$, and 1 year old, $615(\mathrm{n}=3)$ showing subsequent CX3CR1, F4/80 expression for the cells of the CD45 ${ }^{\text {High }}$ gates. Gating of 616 pvM identified as Lyve ${ }^{+} \mathrm{CD} 45^{\text {High }}$ isolated from $C x 3 c r 1^{G F P}$ brain parenchyma. (C) Flow cytometry 617 analysis of CD45 cell subpopulations isolated from Spi $1^{G F P}$ adult brain parenchyma (8-12 weeks after 618 birth, $\mathrm{n}=4$ ), pre-gated on living, single and Lyve ${ }^{+}$cells. Microglia cells were excluded as Lyve1 ${ }^{-}$. 619 Plots show subsequent PU.1, F4/80 expression for the CD45 ${ }^{\text {Int }}$, CD45 ${ }^{\text {Neg }}$ and CD45 ${ }^{\text {High }}$ gates.

Supplementary Figure 4: Gene expression profiling (A) Sort gating strategy for the isolation of the different Lyve1 ${ }^{+}$CD45 populations (B). Single RNA sequencing analysis showing different macrophage associated genes within the tSNE plots.

Supplementary Figure 5: Immunofluorescence of the section of a E18.5 head from a Spi1 ${ }^{G F P /+}$ embryo (PU.1-GFP in green), labeled for Lyve1 (red) and CD45 (white). No GFP or Lyve1 fluorescence was observed in the Spil ${ }^{\text {GFP/GFP }}$ E18.5 superior cortex ( $33 \mu \mathrm{m}$ maximum intensity projection) but we observed Lyve $1^{+}$cells, possibly lymphatic vessels, in the skull. quantify the Lyve1 staining in cleared brains imaged by lightsheet microscopy, Imaris software was used. Using the "Surface tool" of Imaris, a 3D Rendering function, a first volume was created, named «Surface1 » at different locations (Superior cortex and hippocampus) where we wanted to quantify the staining. Based on Lyve1 labelling, a new channel was created. This new channel was used to create a new volume called «Surface 2 » representing the Lyve1 staining. Imaris calculated the different volumes in $\mu \mathrm{m}^{3}$. The ratio « Surface2»/ « Surface1» allows us to normalize the Lyve1 quantification. (B) Gating of conventional pvM identified as Lyve $1^{+} \mathrm{CD} 45^{\text {int }}$ and as Lyve $1^{+} \mathrm{CD} 45^{\text {High }}$ isolated from $\mathrm{CX} 3 \mathrm{crl}^{\text {GFP }}$ brain parenchyma at $\mathrm{P} 14$ after stroke. sagittal half of an adult C57BL/6J mouse brain immunolabeled for Lyvel (red) was acquired on the lightsheet microscope and a 3D reconstruction was made using Imaris. The video zooms in on the 
644 within these regions.

645

646 Supplementary Video 2: Z-stack acquisition by confocal microscopy and 3D reconstruction of 647 the pvM morphology adjacent to a blood vessel within the brain parenchyma. A $100 \mu \mathrm{m}$ brain 648 section was stained with $\alpha$ Lyve1 (red) and $\alpha$ CD31 (green). 3D reconstruction was done using the Imaris 649 surface tool and the video was created using the animation function of Imaris. 


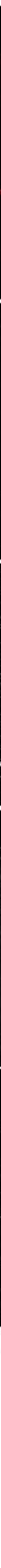




\section{Figure 3}

A P2

Lyve $^{+}$

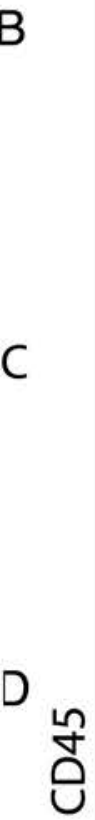

E

\section{F}

P7
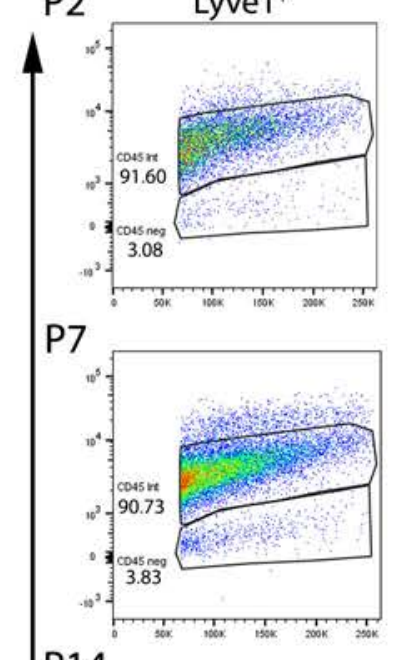

P14

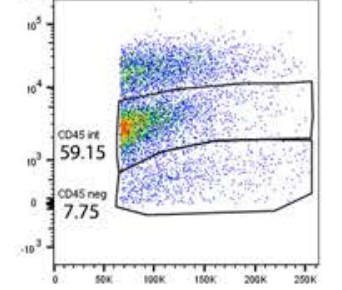

P21

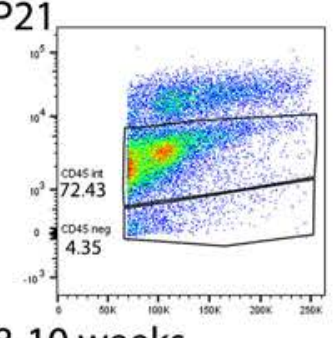

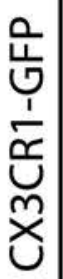

\section{8-10 weeks}

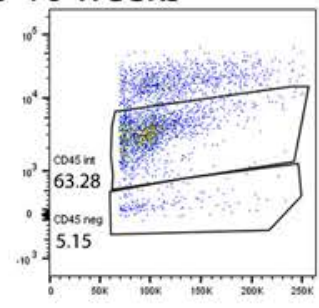

\section{1 year}

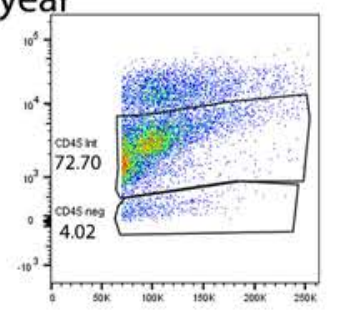

G

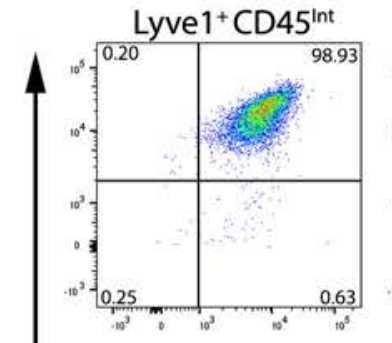

Lyve 1+CD45 ${ }^{\text {Neg }}$
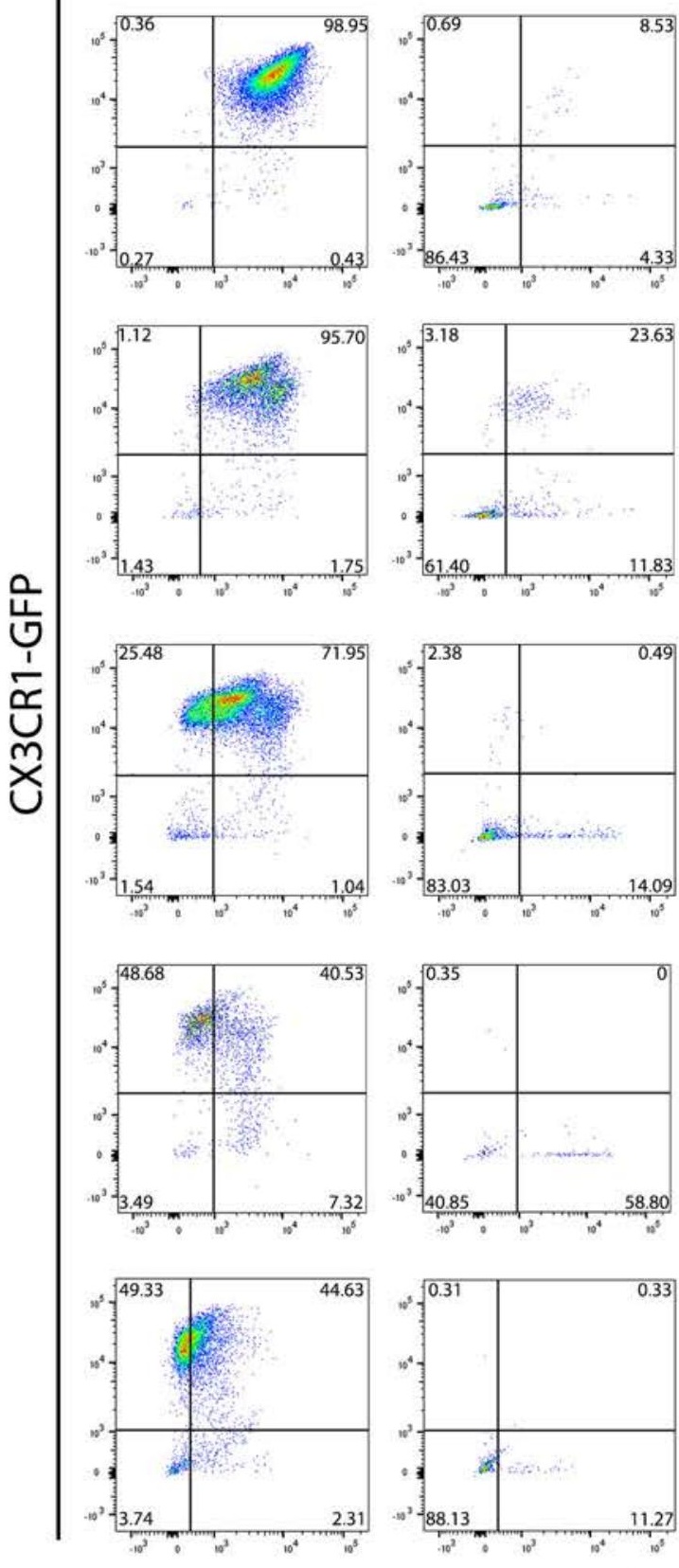

$\mathrm{F} 4 / 80$

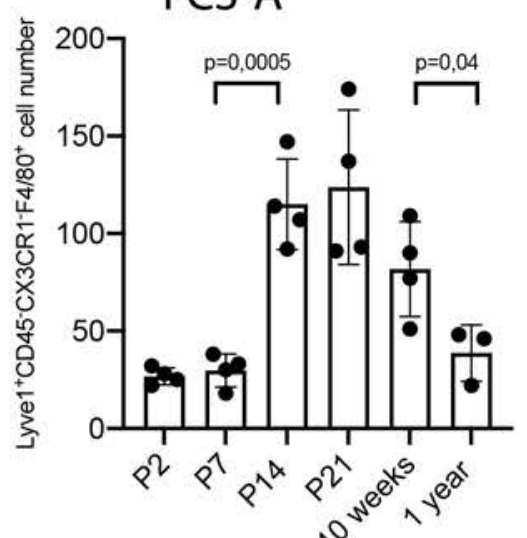


$A$

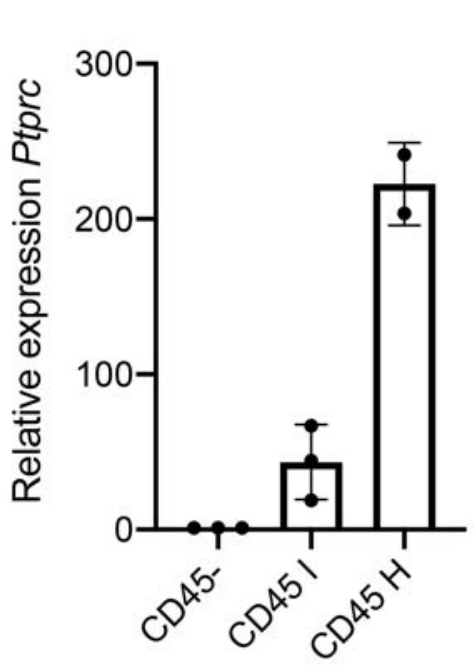

B

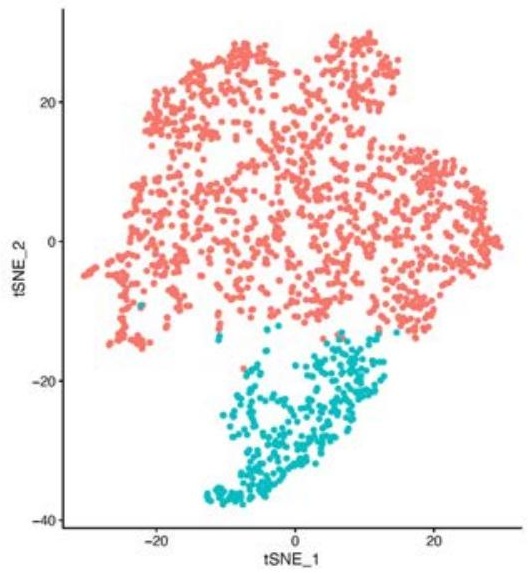

C
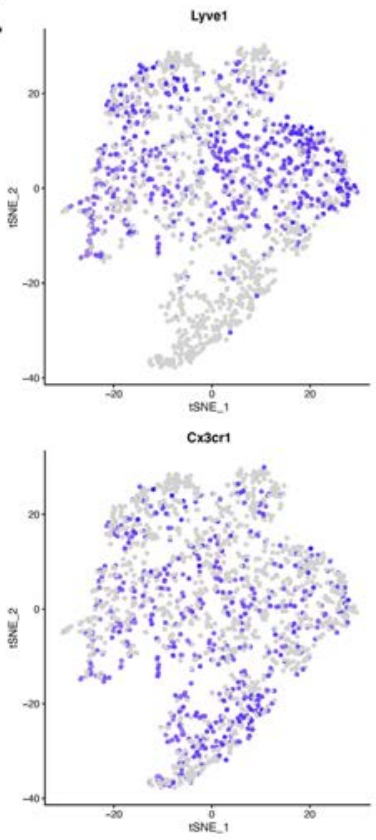
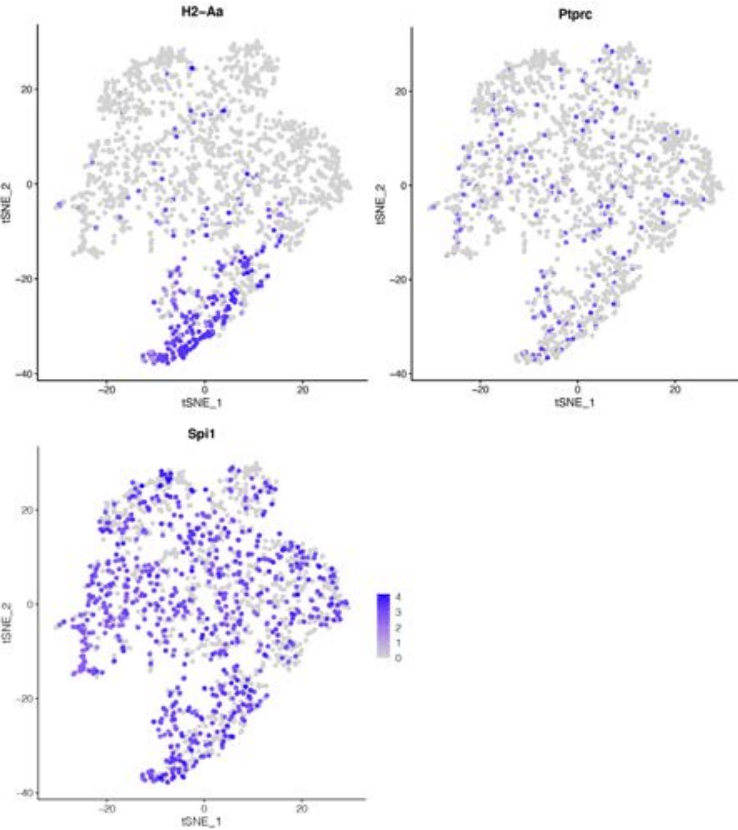

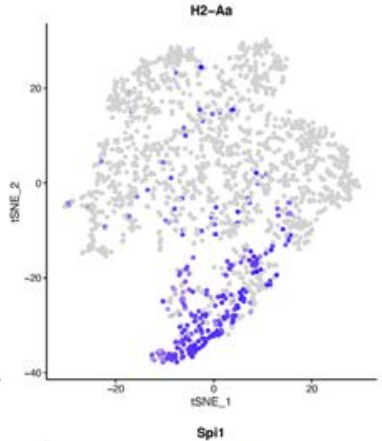


E18.5 Pu1 ${ }^{\mathrm{GFP} /+}$
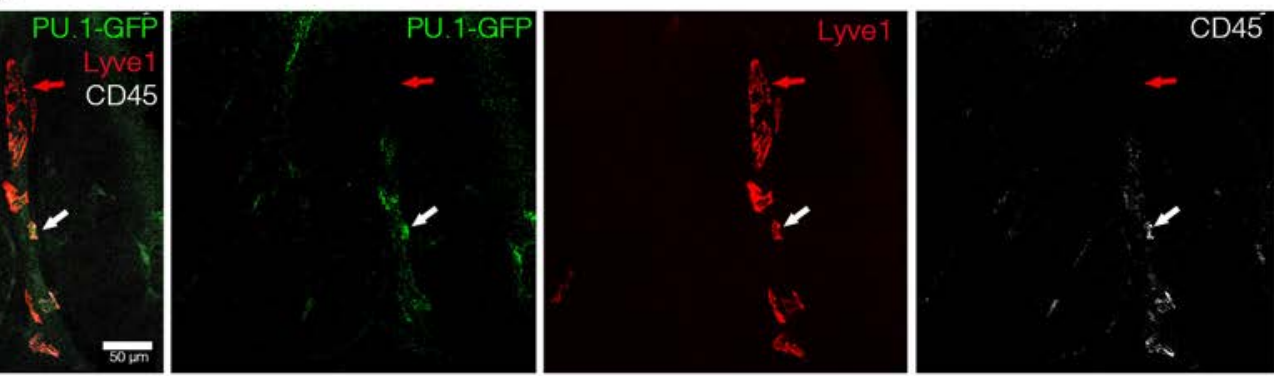

B

E18.5 PU1GFP/GFP
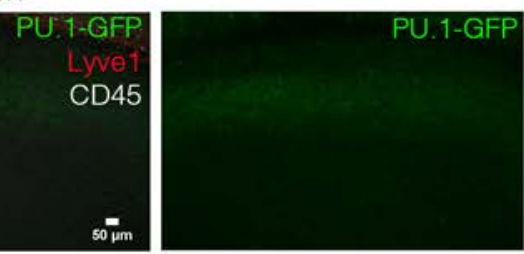

CD45

$\mathrm{C}_{8-12 \text { wk Pu1 }{ }_{\mathrm{GFP} /+}}$

8-12 WU.1-GFP

Superior Cortex CD45
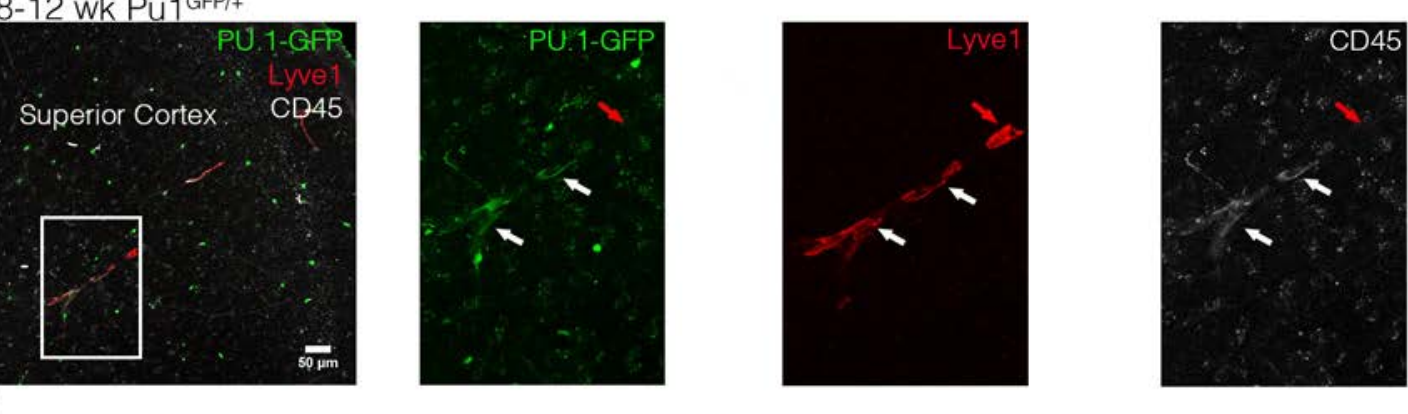

D
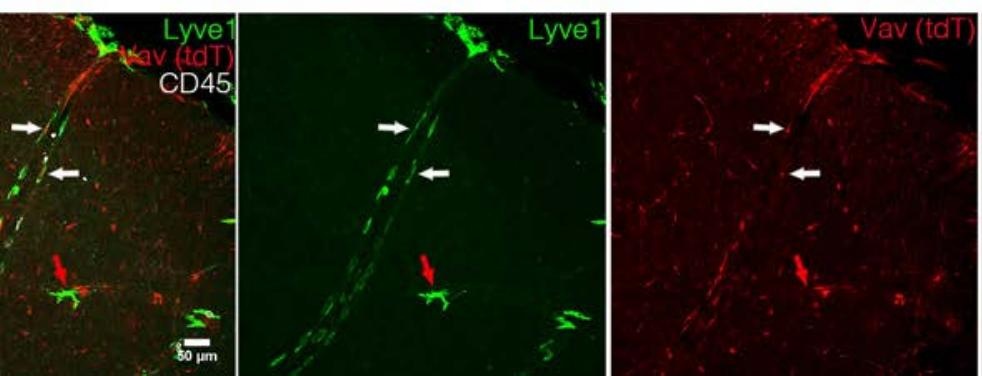

CD45

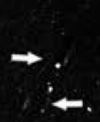




\section{Table 1}

\section{Ab Ressources Table for Imaging (confocal and Lightsheet)}

\begin{tabular}{|l|l|l|}
\hline Primary & & \\
\hline Antibodies & Source & Identifier \\
\hline Lyve-1 & R\&D systems & AF2125 \\
\hline AQP4 & R\&D systems & AB3594 \\
\hline GFAP & Biolegend & 644706 \\
\hline CD45 & eBioscience & $14-0451-85$ \\
\hline MHCII & BD Pharmingen & 562352 \\
\hline F4/80 & Biolegend & 123122 \\
\hline Iba1 & Abcam & ab107159 \\
\hline CD163 & Anders & homemade \\
\hline CD11b & eBioscience & $16-0112-82$ \\
\hline CD206 & Thermofisher & $48-2061-82$ \\
\hline CD31 & Thermo & MA1-40074 \\
\hline Prox-1 & Reliatech & $102-P A 32$ \\
\hline VEGFR3 & R\&D systems & AF743 \\
\hline$\alpha-S M A$ & Thermofisher & $53-9760-82$ \\
\hline PDGFR $\beta$ & Cell Signaling & $3169 S$ \\
\hline ER-T7R & Thermofisher & MA1-40076 \\
\hline CSf-1R & Santa Cruz & sc-692 \\
\hline GFP & AVES & GFP-1020 \\
\hline Laminin 11 & Sorokin L (University of Muenster) & \\
\hline
\end{tabular}

\begin{tabular}{|l|l|l|}
\hline Secondary & & \\
\hline Antibodies & Source & Identifier \\
\hline DaChCy3 & Jackson ImmunoResearch & $703-166-155$ \\
\hline DaG 488 & Thermofisher & A-11055 \\
\hline DaG 555 & Thermofisher & A-21432 \\
\hline DaG 647 & Thermofisher & A-21447 \\
\hline DaG 790 & Jackson ImmunoResearch & $712-655-153$ \\
\hline DaR 488 & Thermofisher & A-21208 \\
\hline DaR 594 & Thermofisher & SA5-10028 \\
\hline DaR 647 & Jackson ImmunoResearch & $712-605-153$ \\
\hline DaRb 488 & Thermofisher & A-21206 \\
\hline DaRb 555 & Thermofisher & A-31572 \\
\hline DaRb 647 & Jackson ImmunoResearch & $711-605-152$ \\
\hline
\end{tabular}


Table 2

\begin{tabular}{|c|c|}
\hline Conventional pvM & pvM2 \\
\hline Lyve $^{+}$ & Lyve1 $^{+}$ \\
\hline $\mathrm{CD} 45^{+}$ & $\mathrm{CD}^{\circ} 5^{-}$ \\
\hline CX3CR1 ${ }^{+}$ & CX3CR1 \\
\hline $\mathrm{PU}^{+}$ & PU1 \\
\hline $\operatorname{Csf1}^{+}$ & Csf1R- $^{-}$ \\
\hline $\mathrm{F} 4 / 80^{+}$ & $\mathrm{F} 4 / 80^{+}$ \\
\hline $\mathrm{Iba1}^{+}$ & $\mathrm{Iba}^{+}$ \\
\hline $\mathrm{CD} 63^{+}$ & CD163 $^{-}$ \\
\hline $\mathrm{CD} 11 \mathrm{~b}^{+}$ & $\mathrm{CD}_{11 b^{-}}$ \\
\hline $\mathrm{CD}^{206^{+}}$ & $\mathrm{CD}^{206^{-}}$ \\
\hline $\mathrm{MHCII}^{+}$ & $\mathrm{MHCll}^{-}$ \\
\hline
\end{tabular}




\section{Supplementary Files}

This is a list of supplementary files associated with this preprint. Click to download.

- pvM2SiretetalSupplFig.pdf

- Video1Lyveinbrainzoominfcortexandhc720.mov

- Video2cd31lyve13dvessel720.mov 